\title{
Stochastic-Geometry-Based Interference Modeling in Automotive Radars Using Matérn Hard-Core Process
}

\author{
Kumar Vijay Mishra, Bhavani Shankar M. R. and Björn Ottersten
}

\begin{abstract}
As the use of radars in autonomous driving systems becomes more prevalent, these systems are increasingly susceptible to mutual interference. In this paper, we employ stochastic geometry to model the automotive radar interference in realistic traffic scenarios and then derive trade-offs between the radar design parameters and detection probability. Prior works model the locations of radars in the lane as a homogeneous Poisson point process (PPP). However, the PPP models assume all nodes to be independent, do not account for the lengths of vehicles, and ignore spatial mutual exclusion. In order to provide a more realistic interference effect, we adopt the Matérn hardcore process (MHCP) instead of PPP, in which two vehicles are not closer than an exclusion radius from one another. We show that the MHCP model leads to more practical design trade-offs for adapting the radar parameters than the conventional PPP model.
\end{abstract}

Keywords-Automotive radar, interference, Matérn hard-core process, Poisson point process, stochastic geometry.

\section{INTRODUCTION}

In recent years, autonomous driving technology has progressed to an initial practical deployment stage [1]. However, substantial challenges remain to ensure its safety and reliability in dense traffic environments [2]. In this context, millimeter-wave (mm-Wave) radar has emerged as an essential sensor that provides important information to the vehicle for recognizing and reacting to traffic conditions in unfavorable conditions such as inclement weather or low visibility [3, 4]. Often vehicles are equipped with multiple radar sensors of varying coverage and diverse functions such as collision avoidance, lane change, blind spot warning, and automatic parking assistance $[5,6]$.

Increased deployment of radars in dense traffic has led to serious challenges of mutual interference and limitation in radar coverage [7-9]. This primarily arises from shared use of limited spectrum in the $77-79 \mathrm{GHz}$ band which leads to not only unintended interference along intersections and highways but also malicious jamming in radar receivers. A brute-force approach of pausing the transmission during the peak interference was common during early days of automotive radar deployment. Now, depending on the transmit waveforms and radar design, a variety of techniques have

K. V. M., B. S. M. R., and B. O. are with the Interdisciplinary Centre for Security, Reliability and Trust (SnT), University of Luxembourg, Luxembourg City L-1855, Luxembourg. E-mail: \{kumar-mishra@ext, bhavani.shankar@, bjorn.ottersten@\}uni.lu.

978-1-7281-8942-0/20/\$31.00 (c)2020 IEEE been proposed to mitigate vehicular radar interference [7]. In general, most of the approaches propose individual vehicles to transmit radar waveforms that are separated in time, frequency, space, and modulation with respect to each other [10]. Among other approaches, digital beamforming is adaptively used in phased array automotive radars to induce spatial separation for eliminating corrupted receive channels [11, 12]. Sometimes, assuming that the interference in the receive signal spectrum is usually stronger, a threshold method is employed [13]. Instead of detecting and canceling the interference, a converse method is proposed in [14] to estimate the target echo in the presence of interference through convex optimization. More recently, techniques to cognitively adapt signal bandwidth and transmit time slot in interference scenarios have been investigated [15].

Nearly all of the above techniques assume deterministic automotive radar interference in a simple two-vehicle topology [16]. This approach excludes stochastic behavior of interference that arises from the spatial distribution of several vehicles on the road. Since the nature of interference is not continuous in space and time, its modeling in realistic traffic environments is helpful in efficient tuning of radar design parameters. In wireless communications, similar problems involving the randomness of emitter locations are usually analyzed using stochastic geometry methods [17]. In addition to modeling the spatial random processes, these techniques often yield mathematical and tractable performance analysis [18]. In this paper, we focus on stochastic geometric modeling of automotive radar interference.

Early works modeled the aggregate interference in a general radar network as Gaussian and derived the probability of detection in the specific case of orthogonal frequency division multiplexing (OFDM) radars [19]. For automotive interference, [20] introduced the stochastic geometric framework to investigate radars in the same lane under different fading conditions using omnidirectional antennas. This was extended for a planar or multi-lane radar network in [21] where vehicles were assumed to be moving in only opposite directions.

Prior works on stochastic-geometry-based interference analyses employ the conventional homogeneous Poisson point process (PPP) for radar locations. This assumption leads to a simplified analysis [22]. However, in practice, the deployment of vehicles is not completely random because any two cars do not drive in very close proximity. This constraint, ignored in PPP with zero correlation, is better captured by non-PPP models such as the Poisson cluster processes [23], Ginibre point processes [24], and hard-core processes (HCP) [25]. In particular, HCP imposes an exclusion ball around every location in the process such that no other vehicle is allowed within this ball of certain radius termed as the hard-core 
distance. However, these models are difficult to analyze and less tractable [26].

In this context, Matérn hard-core process (MHCP) [27] is particularly useful because not only it has a tractable density but it also efficiently reduces the density of points in the parent PPP through the hard-core condition. The MHCP is commonly used for base station models in wireless communications [28] and vehicular [29] communications. It is difficult to analyze these hard-core processes because their probability generating functionals do not exist. In contrast, the clustered models are more tractable. It has also been argued that the nodes further away than the hard-core distance could still be treated as a PPP but it is difficult to verify this analytically [30].

Either of the MHCP Type I or II models are able to generate the homogeneous point processes but only the latter has a higher packing density and is able to approximate inhomogeneous PPP. Both Type I and II are based on a parent PPP of same intensity. However, Type I MHCP silences all nodes with a neighbor within the hard-core distance. This thinning rule is strong but very simple. The Type II MHCP associates a random mark with each node and silences a node only if there is another node within the hard-core distance with a smaller mark. Note that, in point process terminology, the random variables and the resulting random processes are called marks and marked point process. Evidently, the thinning rule for Type II is not very strong. It has been shown that only Type II MHCP causes a level of interference comparable to the one in a PPP [30]. In [31], MHCP - Type II process is used for a vehicular platoon of manual and self-driven vehicles and their effect in the spectral efficient of a vehicle-to-everything (V2X) network is analyzed.

Contrary to prior works which use PPP for automotive radar interference, we consider MHCP - Type II model which takes into account realistic conditions such as the finite length of the vehicle and aggregate interference arising from the minimum distance between the cars. Further, [20, 21] consider the radar cross-section (RCS) of the detected targets to be constant while it is possible to include the fluctuating RCS in our models. The proposed approach is, therefore, more comprehensive in capturing the traffic conditions and radar parameters. This yields valuable trade-offs for designing better automotive radars.

The rest of the paper is organized as follows. In the next section, we introduce the underlying system model. In Section III, we present the statistic of the modeled interference followed by numerical results in Section IV.

\section{SYSTEM MODEL}

Consider a multi-lane road scenario comprising bidirectional traffic (Fig. 1) with yellow arrows indicating the directions. Each vehicle is equipped with a pulsed mm-Wave radar mounted on the front. As the case with mm-Wave band, these radars are mounted with directive antennas having transmit and receive gains $G_{t}$ and $G_{r}$, respectively. The boresight direction of each antenna is an independent and uniform random variable in $[0,2 \pi)$.

Assuming a simple case when the vehicles in each direction are situated completely independent of each other, we model

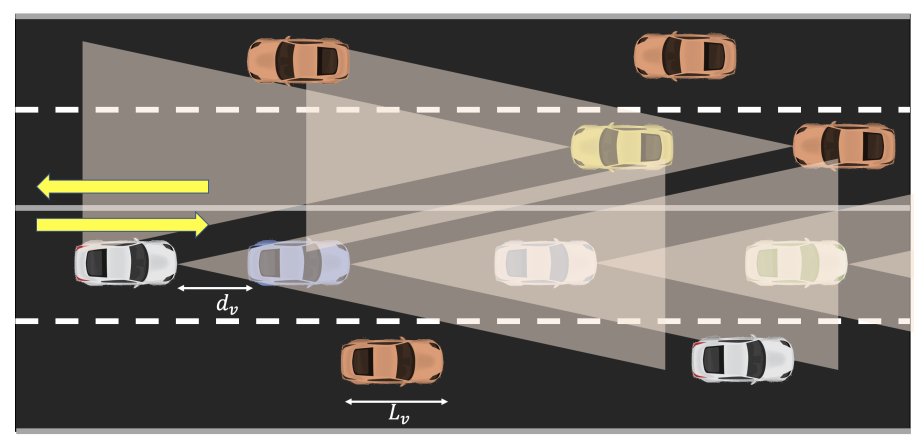

Fig. 1. A simplified illustration of the geometry of bidirectional, multi-lane traffic scenario. Yellow arrows indicate the direction of traffic in the respective lanes. Each vehicle is mounted with the radar whose approximate coverage is indicated by the gray triangular area. The interference to a typical vehicle (yellow) is mainly caused by approaching vehicles driving in the opposite direction. The maximum length of each vehicle is $L_{v}$ while any two vehicles in the lane are separated by a minimum distance $d_{v}$.

their locations as a homogeneous PPP $\Phi=\left\{\mathbf{x}_{i}\right\}$ of density $\lambda$ over $\mathbb{R}^{2}$. Each radar transmits constant power $P_{t}$ at a pulse repetition frequency (PRF) $f_{p}=1 / T_{p}$, where we consider the pulse repetition interval (PRI) $T_{p}=M \tau$ to be a multiple of pulse-width $\tau$. As in other approaches, it will be optimum for the radars to synchronize at slot-level such that all radars follow a cycle using one slot out of $M$ at a time [15]. Then, the receive duration consists of $M-1$ slots. However, the vehicles are in general not coordinated. This lack of synchronization is modeled by introducing random offsets in the operating cycles of different radars. Accordingly, each PPP element is assigned an independent mark $m_{i} \sim \mathcal{U}\{0, M-1\}$ and transmits at slots $m_{i}+k M, k \in \mathbb{N}$.

The interference for a typical vehicle (yellow car in Fig. 1) arises mainly from the transmission of the approaching vehicles in the opposite direction. We assume there are $K$ lanes in the opposite direction. Without loss of generality, assume the typical vehicle is located at the origin of the plane. We consider a simple signal-threshold detector such that a target is declared present if the cumulative received signal strength over any of the $M-1$ slots for the echo exceeds a threshold $\gamma$. The path loss for signal propagation has exponent $\alpha>2$ [4]. For a target of constant radar cross section $\sigma$ in the boresight direction of the typical node, the received power $\mathcal{S}$ is

$$
P_{r}=\frac{P_{t} G_{t} G_{r} A_{r} \sigma l}{4 \pi} \cdot \zeta d^{-2 \alpha}
$$

Here, $l=\left(c /\left(4 \pi f_{c}\right)\right)^{2}, c=3 \times 10^{8} \mathrm{~m} / \mathrm{s}$ is the speed of light, $f_{c}$ is the carrier frequency, $A_{r}$ is the receiver processing gain, $d$ is the target range, and $\zeta$ is the small-scale fading coefficient that is unity in the absence of fading and $\sim \exp (1)$ for Rayleigh fading [4].

In a practical traffic scenario, the vehicles are not distributed as PPP. If the maximum length of each vehicle is $L_{v}$ and, in compliance with safety regulations, any two vehicles in the $k$-th lane keep a mutual separation of $d_{v_{k}}=d_{v}$, the hard-core distance is $d_{h_{k}}=d_{v}+L_{v}$. Then, from the initial PPP $\Phi$, where each point $\mathbf{x}_{i} \in \Phi$ has an independent mark $m_{i}$, we obtain 


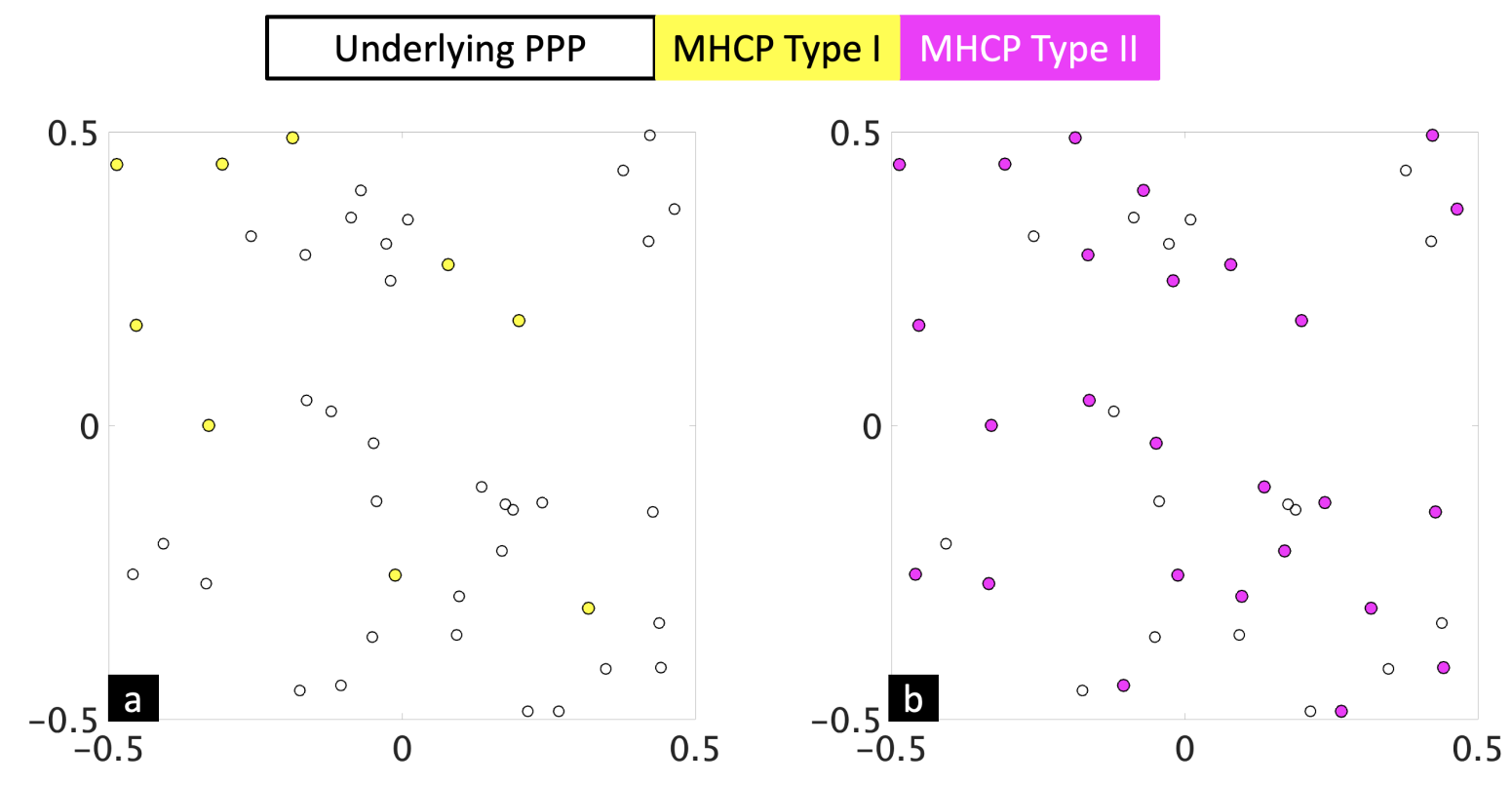

Fig. 2. (a) Type I and (b) Type II MHCP plotted along with the same underlying PPP with $\lambda=50$ and $d_{h_{k}}=0.1$.

Type II MHCP as follows. We select the point $\mathbf{x}_{i} \in \Phi$ if it has the lowest mark among all the points in the ball $\mathcal{B}\left(\mathbf{x}_{i}, d_{h_{k}}\right)$, i.e.,

$$
\Phi_{\mathcal{I}_{k}}=\left\{x_{j}: x_{j} \in \Phi, m_{j} \leq m_{i}, \forall \mathbf{x}_{i} \in\left(\mathcal{B}\left(\mathbf{x}_{i}, d_{h_{k}}\right) \cap \Phi\right)\right\} .
$$

We remove all flagged points and the remaining points form a MHCP $\Phi_{\mathcal{M}_{k}}$ for the $k$-th lane. The density of $\Phi_{\mathcal{M}_{k}}$ is [27]

$$
\lambda_{\mathcal{M}_{k}}=\frac{1-\exp \left(-\lambda \pi d_{h_{k}}^{2}\right)}{\pi d_{h_{k}}^{2}}
$$

where $\lambda \pi d_{h_{k}}^{2}$ is simply the area of the core. Note that, in case of Type I MHCP $\Phi_{\mathcal{Q}_{k}}$, the density is

$$
\lambda_{\mathcal{Q}_{k}}=\lambda \exp \left(-\lambda \pi d_{h_{k}}^{2}\right),
$$

so that

$$
\lambda_{\mathcal{M}_{k}}=\frac{1}{\pi d_{h_{k}}^{2}}\left(1-\frac{\lambda_{\mathcal{Q}_{k}}}{\lambda}\right) .
$$

Figure 2 shows the difference in thinning obtained via both processes. On a two-dimensional plane $[-0.5,0.5] \times$ $[-0.5,0.5]$, we generated the underlying PPP with density $\lambda=50$. Then, setting the radius of hard-core $d_{h_{k}}=0.1$, we obtain Type I and Type II MHCPs. The strong thinning in MHCP Type I is evident.

The aggregate interference $I$ that the typical radar receives during each of the $M-1$ detection slots is independent and identically distributed. Summing the contributions of all lanes, we get

$$
I=\sum_{k=1}^{K} \sum_{\mathbf{x} \in \Phi_{\mathcal{M}_{k}}} P_{t} G_{t} G_{r} l \zeta\|\mathbf{x}\|^{-\alpha} .
$$

For the threshold detector, a target is correctly detected with detection probability

$$
P_{d}=\mathbb{P}\left\{P_{r}+I+N \geq \gamma\right\},
$$

where $N$ represents the power of random noise at the receiver. The threshold detection is a common technique adopted in practical radar receivers [32] but these energy detectors could be sensitive to thresholds.

However, the interference $I \gg N$ and therefore we neglect the contribution from noise. Similarly, the radar yields a false alarm when the power over any one of the receive slots is greater than the threshold while the target was absent. The probability of false alarm is

$$
P_{f a}=1-(\mathbb{P}\{I \leq i\})^{M-1}=1-F_{I}(\gamma)^{M-1},
$$

where $F_{\mathcal{I}}(i)$ is the cumulative distribution function (CDF) of $I$ and we assumed independence across different slots. Operationally, the threshold $\gamma$ is selected such that a constant false alarm rate (CFAR) is maintained and then corresponding $P_{d}$ is evaluated.

\section{INTERFERENCE STATISTICS}

It is clear from (7) that detection performance depends on accurate statistical modeling of the interference $I$. For omnidirectional beams, conventional stochastic geometry provides closed form expressions for only $\alpha=4$ by modeling $I$ as Lévy distributed. In order to derive useful expressions for general $\alpha$, [21] tuned $\gamma$ based on the power distribution of only the strongest interferer. The antenna pattern is assumed as an ideal cone with gains $G_{t}$ and $G_{r}$ over a beamwidth $\varphi$.

A typical vehicle observes interference in its slot only if the alignment of antenna beam from the other vehicle will 
overlap with its own. Therefore, the probability of this event is $\varphi^{2} /\left(4 \pi^{2}\right)$. This further reduces the density from $\lambda_{\mathcal{M}_{k}}$ to $\lambda_{\mathcal{M}_{k}} \varphi^{2} /\left(4 \pi^{2}\right)$. If $I_{s}$ is the power of the strongest interferer, then following [21] for this new density, the cone antenna pattern assumption gives

$$
F_{I_{s}}(i)=\mathbb{P}\left\{I_{s} \leq i\right\}=\exp \left(-\frac{\lambda_{\mathcal{M}_{k}} f_{p} \varphi^{2} \Omega \omega^{2 / \alpha}}{4 \pi} \cdot i^{-2 / \alpha}\right)
$$

where $\omega=P_{t} G_{t} G_{r} l, \Omega=1$ in the absence of fading and $\Omega=$ $\Gamma(1+2 / \alpha)$ for Rayleigh fading, with $\Gamma(x)=\int_{0}^{\infty} x^{t-1} e^{-x} d t$. Then, the probability of false alarm is simply $P_{f a}=1-$ $F_{I_{s}}(\gamma)^{M-1}$ and

$$
\gamma=\omega\left(\frac{-\Omega\left(1-f_{p}\right) \lambda_{\mathcal{M}_{k}} \varphi^{2}}{4 \pi \ln \left(1-P_{f a}\right)}\right)^{\alpha / 2}
$$

In the absence of fading, the probability of detection is $P_{d}=1-F_{I_{s}}\left(\gamma-P_{r}\right)$. Then, a target is detected with probability 1 if it is near enough to satisfy the condition $P_{r}>\gamma$. As $P_{r} \rightarrow 0$, a detection occurs with probability $\mathbb{P}\{I>\gamma\}=1-\left(1-P_{f a}\right)^{1 /(M-1)}$. In mm-Wave channel, signals undergo multiple reflections. We model this behavior by setting a higher path-loss exponent and considering Rayleigh fading on the interference and target signals. The detection probability is then conditioned on the interference power as $P_{d}=\mathbb{E}_{I}\left[\mathbb{P}\left\{\zeta \geq(\gamma-I) 4 \pi d^{2 \alpha} /\left(\omega A_{r} \sigma\right) \mid I\right\}\right]$, with $\zeta \sim \exp (1)$. For the strongest interferer approximation and cone antenna pattern, this becomes

$$
P_{d}=1-F_{I_{s}}(\gamma)+\int_{0}^{\gamma} e^{-\frac{(\gamma-i) 4 \pi d^{2 \alpha}}{\omega A_{r} \sigma}} f_{I_{s}}(i) d i
$$

where $f_{I_{s}}(i)=d F_{I_{s}} / d i$ is the probability density function (PDF) and $1-F_{I_{s}}(\gamma)$ corresponds to the event when interference is above the threshold, i.e. a target is present regardless of the strength of $P_{r}$. The detection rate is now calculated using numerical integration

Finally, for the multi-lane case, we label the left side of (9) as $F_{I_{s}, k}(i)$. The new

$$
F_{I_{s}}(i)=\left(F_{I_{s}, 1}(i) * F_{I_{s}, 2}(i) * \cdots * F_{I_{s}, K}(i)\right)^{\prime},
$$

where $*$ is the convolution oepration and $(\cdot)^{\prime}$ indicates the derivative. Note that the statistics of the RCS $\sigma$ could be incorporated in these expressions.

\section{EXPERIMENTS}

We evaluated our method for the most general case of Rayleigh fading and multiple lanes. The transmit power was set to $P_{t}=10 \mathrm{dBm}$ with $G_{t}=G_{r}=30 \mathrm{dBi}, f_{c}=77 \mathrm{GHz}$, $\varphi=\pi / 6, \alpha=3$ and $\tau=0.4 \mu \mathrm{s}$. Then, for $\gamma=5 \mathrm{~dB}$, constant $\sigma=30 \mathrm{dBsm}$ and $K=5$ lanes, the probability of detection is plotted in Fig. 3 as a function of range. Both MonteCarlo (MC) and numerical solutions are compared for various original PPP densities. The decline in the $P_{d}$ is precipitous with increasing range. Note that as the density increases, the radar encounters more interference leading to a sharp fall in detection performance. In future, our goal is to generalize this model for other RCS types.

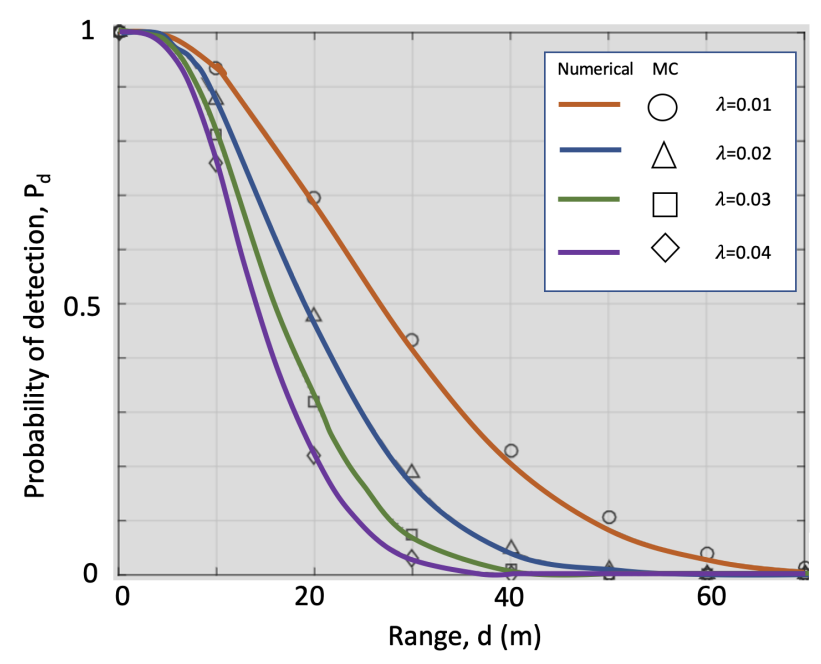

Fig. 3. Probability of detection for the Rayleigh fading case and five lanes.

\section{REFERENCES}

[1] H. Hartenstein and L. Laberteaux, "A tutorial survey on vehicular ad hoc networks," IEEE Communications Magazine, vol. 46, no. 6, pp. 164-171, 2008.

[2] N. Lu, N. Cheng, N. Zhang, X. Shen, and J. W. Mark, "Connected vehicles: Solutions and challenges," IEEE Internet of Things Journal, vol. 1, no. 4, pp. 289-299, 2014.

[3] Z. Slavik and K. V. Mishra, "Phenomenological modeling of millimeterwave automotive radar," in URSI Asia-Pacific Radio Science Conference, 2019, pp. 1-4.

[4] K. V. Mishra, M. R. Bhavani Shankar, V. Koivunen, B. Ottersten, and S. A. Vorobyov, "Toward millimeter wave joint radar communications: A signal processing perspective," IEEE Signal Processing Magazine, vol. 36, pp. 100-114, 2019.

[5] G. Duggal, S. Vishwakarma, K. V. Mishra, and S. S. Ram, "Dopplerresilient 802.11ad-based ultra-short range automotive joint radarcommunications system," IEEE Transactions on Aerospace and Electronic Systems, 2020, in press.

[6] I. Bilik, O. Longman, S. Villeval, and J. Tabrikian, "The rise of radar for autonomous vehicles: Signal processing solutions and future research directions," IEEE Signal Processing Magazine, vol. 36, no. 5, pp. 2031, 2019.

[7] S. Alland, W. Stark, M. Ali, and M. Hegde, "Interference in automotive radar systems: Characteristics, mitigation techniques, and current and future research," IEEE Signal Processing Magazine, vol. 36, no. 5, pp. 45-59, 2019.

[8] H. Bloecher and J. Dickmann, "Automotive radar sensor interference Thread and probable countermeasures," in International Radar Symposium, 2018, pp. 1-7.

[9] A. Ayyar and K. V. Mishra, "Robust communications-centric coexistence for turbo-coded OFDM with non-traditional radar interference models," in IEEE Radar Conference, 2019, pp. 1-6.

[10] C. Aydogdu, G. K. Carvajal, O. Eriksson, H. Hellsten, H. Herbertsson, M. F. Keskin, E. Nilsson, M. Rydström, K. Vanäs, and H. Wymeersch, "Radar interference mitigation for automated driving," arXiv preprint arXiv:1909.09441, 2019.

[11] M. Goppelt, H. Bloecher, and W. Menzel, "Automotive radar - Investigation of mutual interference mechanisms," Advances in Radio Science, vol. 8, no. B. 3, pp. 55-60, 2010.

[12] M. Rameez, M. Dahl, and M. I. Pettersson, "Adaptive digital beam- 
forming for interference suppression in automotive FMCW radars," in IEEE Radar Conference, 2018, pp. 252-256.

[13] B. Nuss, L. Sit, and T. Zwick, "A novel technique for interference mitigation in OFDM radar using compressed sensing," in IEEE International Conference on Microwaves for Intelligent Mobility, 2017, pp. 143-146.

[14] F. Uysal and S. Sanka, "Mitigation of automotive radar interference," in IEEE Radar Conference, 2018, pp. 405-410.

[15] Z. Slavik and K. V. Mishra, "Cognitive interference mitigation in automotive radars," in IEEE Radar Conference, 2019, pp. 1-6.

[16] G. M. Brooker, "Mutual interference of millimeter-wave radar systems," IEEE Transactions on Electromagnetic Compatibility, vol. 49, no. 1, pp. 170-181, 2007.

[17] M. Rebato, J. Park, P. Popovski, E. De Carvalho, and M. Zorzi, "Stochastic geometric coverage analysis in mmwave cellular networks with realistic channel and antenna radiation models," IEEE Transactions on Communications, vol. 67, no. 5, pp. 3736-3752, 2019.

[18] M. Haenggi, Stochastic geometry for wireless networks. Cambridge University Press, 2012

[19] M. Braun, R. Tanbourgi, and F. K. Jondral, "Co-channel interference limitations of OFDM communication-radar networks," EURASIP Journal on Wireless Communications and Networking, vol. 2013, no. 1, p. 207, 2013.

[20] A. Al-Hourani, R. J. Evans, S. Kandeepan, B. Moran, and H. Eltom, "Stochastic geometry methods for modeling automotive radar interference," IEEE Transactions on Intelligent Transportation Systems, vol. 19, no. 2, pp. 333-344, 2017.

[21] A. Munari, L. Simić, and M. Petrova, "Stochastic geometry interference analysis of radar network performance," IEEE Communications Letters, vol. 22, no. 11, pp. 2362-2365, 2018.

[22] H. S. Dhillon, R. K. Ganti, F. Baccelli, and J. G. Andrews, "Modeling and analysis of K-tier downlink heterogeneous cellular networks," IEEE Journal on Selected Areas in Communications, vol. 30, no. 3, pp. 550$560,2012$.
[23] C. Saha, M. Afshang, and H. S. Dhillon, "3GPP-inspired HetNet model using Poisson cluster process: Sum-product functionals and downlink coverage," IEEE Transactions on Communications, vol. 66, no. 5, pp. 2219-2234, 2017.

[24] N. Deng, W. Zhou, and M. Haenggi, "The Ginibre point process as a model for wireless networks with repulsion," IEEE Transactions on Wireless Communications, vol. 14, no. 1, pp. 107-121, 2014.

[25] I. Flint, H.-B. Kong, N. Privault, P. Wang, and D. Niyato, "Analysis of heterogeneous wireless networks using Poisson hard-core hole process," IEEE Transactions on Wireless Communications, vol. 16, no. 11, pp. 7152-7167, 2017.

[26] A. Al-Hourani, R. J. Evans, and S. Kandeepan, "Nearest neighbor distance distribution in hard-core point processes," IEEE Communications Letters, vol. 20, no. 9, pp. 1872-1875, 2016.

[27] J. Teichmann, F. Ballani, and K. G. van den Boogaart, "Generalizations of Matérn's hard-core point processes," Spatial Statistics, vol. 3, pp. 33-53, 2013.

[28] M. Di Renzo, A. Zappone, T. T. Lam, and M. Debbah, "System-level modeling and optimization of the energy efficiency in cellular networks - A stochastic geometry framework," IEEE Transactions on Wireless Communications, vol. 17, no. 4, pp. 2539-2556, 2018.

[29] F. J. Martin-Vega, B. Soret, M. C. Aguayo-Torres, I. Z. Kovacs, and G. Gomez, "Geolocation-based access for vehicular communications: Analysis and optimization via stochastic geometry," IEEE Transactions on Vehicular Technology, vol. 67, no. 4, pp. 3069-3084, 2017.

[30] M. Haenggi, "Mean interference in hard-core wireless networks," IEEE Communications Letters, vol. 15, no. 8, pp. 792-794, 2011.

[31] W. Yi, Y. Liu, Y. Deng, A. Nallanathan, and R. W. Heath, "Modeling and analysis of MmWave V2X networks with vehicular platoon systems," IEEE Journal on Selected Areas in Communications, vol. 37, no. 12, pp. 2851-2866, 2019.

[32] S. M. Kay, Fundamentals of statistical signal processing: Detection theory. Prentice Hall, 1993. 